\title{
TOTALLY ZIPPIN $p$-GROUPS
}

\author{
CHARLES MEGIBBEN
}

\begin{abstract}
If $G$ is a $p$-group of limit length $\lambda$, then it satisfies the $\lambda$-Zippin property provided that whenever $A / p^{\lambda} A \cong G \cong B / p^{\lambda} B$, every isomorphism between $p^{\lambda} A$ and $p^{\lambda} B$ extends to an isomorphism between $A$ and $B$. We show that if $G$ is almost balanced in a totally projective group, then $G$ does satisfy the $\lambda$-Zippin property. This leads to the existence of a great variety of $G$ 's that are totally Zippin in the sense that $G / p^{\alpha} G$ satisfies the $\alpha$-Zippin property for all limit ordinals $\alpha \leq \lambda=$ length of $G$. Hence totally Zippin $p$-groups need not be $S$-groups, although those of countable length turn out to be direct sums of countable groups.
\end{abstract}

Let $G$ be an abelian $p$-group of limit length $\lambda$. A $p$-group $A$ will be called a $\lambda$ elongation by $G$ if $A / p^{\lambda} A \cong G$. Following Warfield [13], we say that $G$ satisties the $\lambda$-Zippin property if the following holds: If $A$ and $B$ are $\lambda$-elongations by $G$, then every isomorphism between $p^{\lambda} A$ and $p^{\lambda} B$ extends to an isomorphism between $A$ and $B$. Zippin proved that $G$ has this property if it is countable and Hill generalized this to the class of totally projective $p$-groups. In fact, Nunke [8] showed that the same is true for $S$-groups (in the sense of Warfield [11], and that furthermore only the $\Sigma$-cyclic $p$-groups enjoy the $\omega$-Zippin property. For $\lambda>\omega$, however, examples in [8] suggest that nothing very definitive can be established about the structure of $p$-groups with the $\lambda$-Zippin property. Consequently, Warfield $[\mathbf{1 3}]$ proposed as a more tractable class the $G$ 's that are totally Zippin in the sense that $G / p^{\alpha} G$ has the $\alpha$-Zippin property for all limit ordinals $\alpha \leq \lambda$. He moreover suggested that a reasonable conjecture would be that the totally Zippin $p$-groups are precisely the $S$-groups. This turns out not to be so and we shall, in fact, establish the existence of a considerable variety of totally Zippin $p$-groups of length $\Omega=\omega_{1}$. On a more positive note, we show that when $\lambda$ is a countable limit ordinal, the only totally Zippin $p$-groups of length $\lambda$ are the d.s.c.'s (direct sums of countable reduced $p$ groups); that is, Warfield's conjecture does hold for groups of countable length.

Call a subgroup $H$ of $G$ almost balanced if it is isotype (i.e., $p^{\alpha} G \cap H=p^{\alpha} H$ for all ordinals $\alpha$ ) and $p^{\alpha}(G / H)=p^{\alpha} G+H / H$ for all $\alpha<\lambda=$ length of $G$. The isotype and $\lambda$-dense $\left(G=p^{\alpha} G+H\right.$ for all $\left.\alpha<\lambda\right)$ subgroups $H$ of $G$ are just the almost balanced subgroups with $G / H$ divisible. As the following technical result indicates, this latter class of subgroups plays a crucial role in the study of $\lambda$-elongations by $G$.

Proposition. Suppose $A$ is a $\lambda$-elongation by $G$ where $\lambda$ is a limit ordinal and let $D$ be the divisible hull of $p^{\lambda} A$. Then there is an isomorphism $\theta: G / H \rightarrow D / p^{\lambda} A$,

Received by the editors February 24, 1983.

1980 Mathematics Subject Classification. Primary 20K10, 20K25, 20K35; Secondary $20 \mathrm{~K} 40$.

Key words and phrases. $p$-groups, totally projective, $\lambda$-elongation, totally Zippin, $S$-groups, d.s.c, $C_{\lambda}$-groups. 
where $H$ is a $\lambda$-dense, isotype subgroup of $G$ and $A$ can be realized as the subgroup of $G \oplus D$ consisting of all pairs $(g, d)$, where $\theta(g+H)=d+p^{\lambda} A$. Conversely, any $A$ so realized will be a $\lambda$-elongation by $G$.

The foregoing result evidently dates back to Kulikov $[\mathbf{3}]$ and the proof given in [5] for the case $\lambda=\omega$ readily generalizes.

We say that subgroups $H$ and $K$ of $G$ are congruent over $G$ provided $\psi(H)=K$ for some automorphism $\psi$ of $G$. If furthermore for each isomorphism $\mu: G / H \rightarrow$ $G / K$ there is such an automorphism inducing $\mu$, then we say that $H$ and $K$ are strongly congruent over $G$.

THEOREM 1. Let $G$ be a p-group of limit length $\lambda$. If each pair of $\lambda$-dense, isotype subgroups $H$ and $K$ with $G / H \simeq G / K$ are strongly congruent over $G$, then $G$ satisfies the $\lambda$-Zippin property

Proof. Suppose $A$ and $B$ are $\lambda$-elongations by $G$ and let $\phi: p^{\lambda} A \rightarrow p^{\lambda} B$ be a fixed isomorphism. Without loss of generality, we may assume that $p^{\lambda} A$ and $p^{\lambda} B$ have a common divisible hull $D$ and extend $\phi$ to an automorphism $\phi^{\prime}$ of $D$. By our Proposition we have a pair of $\lambda$-dense, isotype subgroups $H$ and $K$ of $G$ and a pair of isomorphisms $\theta_{A}: G / H \rightarrow D / p^{\lambda} A$ and $\theta_{B}: G / K \rightarrow D / p^{\lambda} B$ yielding representations of $A$ and $B$ as subgroups of $G \oplus D$. Now consider the diagram

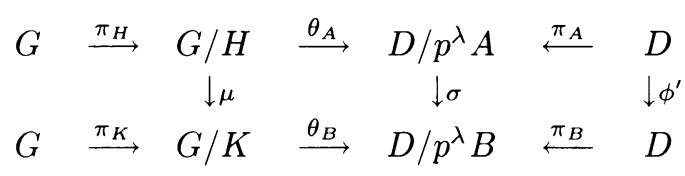

where $\sigma$ is induced by $\phi^{\prime}$, the $\pi$ 's are canonical and $\mu=\theta_{B}^{-1} \sigma \theta_{A}$. By our hypothesis on $G$, we have an automorphism $\psi$ of $G$ such that $\pi_{K} \psi=\mu \pi_{H}$. Then $\xi=\psi \oplus \phi^{\prime}$ is an automorphism of $G \oplus D$ and we claim that $\xi$ maps $A$ onto $B$. Indeed if $a=(g, d) \in A$, then $\theta_{A} \pi_{H}(g)=\pi_{A}(d)$ and therefore

$$
\theta_{B} \pi_{K} \psi(g)=\theta_{B} \mu \pi_{H}(g)=\sigma \theta_{A} \pi_{H}(g)=\theta \pi_{A}(d)=\pi_{B} \phi^{\prime}(d) ;
$$

that is, $\xi(a)=\left(\psi(g), \phi^{\prime}(d)\right)$ is in $B$. By symmetry, $\xi^{-1}$ maps $B$ to $A$ and hence $\xi$ maps $A$ onto $B$.

To give some substance to Theorem 1, we quote from $[\mathbf{2}]$ the following result: If $H$ and $K$ are almost balanced subgroups of the totally projective $p$-group $G$ of limit length, where $H$ and $K$ have the same Ulm invariants and $G / H \cong G / K$, then $H$ and $K$ are strongly congruent over $G$. This observation leads rather easily to

THEOREM 2. If $G$ has limit length $\lambda$ and is an almost balanced subgroup of a totally projective p-group, then $G$ satisfies the $\lambda$-Zippin property.

PROOF. Let $G$ be almost balanced in the totally projective $p$-group $M$. Replacing $M$ by $M / p^{\lambda} M$ if necessary, we may assume that $\lambda$ is also the length of $M$. Now let $H$ and $K$ be $\lambda$-dense, isotype subgroups of $G$ with $G / H \cong G / K$. Since the property of being almost balanced is transitive, $H$ and $K$ are almost balanced in $M$. Take $\mu: G / H \rightarrow G / K$ to be any isomorphism from $G / H$ onto $G / K$. Since $G / H$ and $G / K$ are divisible, they are summands of $M / H$ and $M / K$, respectively, with complements isomorphic to $M / G$. Therefore $\mu$ extends to an isomorphism 
$\mu^{\prime}: M / H \rightarrow M / K$. By the Hill-Megibben theorem quoted above, there is an automorphism $\psi^{\prime}$ of $M$ that induces $\mu^{\prime}$. But since $\mu^{\prime}$ extends $\mu$, it follows that $\psi^{\prime}$ maps $G$ onto itself; that is, $\psi^{\prime}$ restricts to an automorphism $\psi$ of $G$ that induces $\mu$ and hence $H$ and $K$ are strongly congruent over $G$.

THEOREM 3. If $G$ has limit length and is almost balanced in a direct sum of countable reduced p-groups, then $G$ is a totally Zippin p-group

Proof. Let $G$ be almost balanced in the d.s.c. $M$. By a well-known theorem of Hill's, $G / p^{\alpha} G$ is a d.s.c. for all $\alpha<\Omega$. In particular, if $G$ has countable length, then it is a d.s.c. and hence totally Zippin. On the other hand, if $G$ has length $\Omega$, then it satisfies the $\Omega$-Zippin property by Theorem 2 and hence is totally Zippin by our above observation on the quotients $G / p^{\alpha} G$.

The foregoing theorem yields a great variety of totally Zippin $p$-groups that fail to be $S$-groups. First observe by results in [7] that every abelian $p$-group is realizable as a quotient $M / G$ where $M$ is a d.s.c. of length $\Omega$ and $G$ is almost balanced in $M$. If $p^{\Omega}(M / G)$ is reduced and nonzero, then $G$ cannot be an $S$-group. This embarrassment of riches is, of course, due to $\Omega$ being the first limit ordinal not cofinal with $\omega$. Matters are much simpler for ordinals cofinal with $\omega$ and, as observed earlier, we shall show there are no totally Zippin $p$-groups of countable length other than the d.s.c.'s. In fact if $G$ has length $\lambda$ cofinal with $\omega$ and if $G$ is a $C_{\lambda}$-group (that is, $G / p^{\alpha} G$ is totally projective for all $\alpha<\lambda$ ), then $G$ will satisfy the $\lambda$-Zippin property only if it is totally projective. Indeed using a recent theorem of Hill and the theory of $C_{\lambda}$-groups, we can establish this by generalizing Warfield's proof in $[\mathbf{1 2}]$ that only $\Sigma$-cyclic $p$-groups satisfy the $\omega$-Zippin property.

THEOREM 4. Let $G$ be a $C_{\lambda}$-group of length $\lambda$, where $\lambda$ is cofinal with $\omega$. Then $G$ satisfies the $\lambda$-Zippin property if and only if $G$ is totally projective.

Proof. Assume that $G$ is not totally projective. We shall show that there exist nonisomorphic $\lambda$-elongations $A$ and $B$ of $G$, where $p^{\lambda} A \cong p^{\lambda} B$. By Theorem 2.7 in $[\mathbf{1 0}], G$ contains a $\lambda$-dense, isotype subgroup $H$ such that $H$ is totally projective. Since $G$ is not totally projective, the divisible group $G / H$ is necessarily uncountable (see Theorem 1 in $[\mathbf{9}]$ ). Therefore we have a direct decomposition $G / H=\bigoplus_{n=1}^{\infty} H_{n} / H$, where $H_{n} / H \cong G / H$ for all $n$. Then $G$ is the union as an ascending sequence $\left\{G_{n}\right\}_{n<\omega}$ of $\lambda$-dense, isotype subgroups where $G_{0}=H, G_{n} / H=\bigoplus_{k<n} H_{k} / H$ and $G / G_{n} \cong G / H$ for all $n$. By Theorem 1 in $[\mathbf{1}]$, not all of the $G_{n}$ 's can be totally projective. Thus $G$ contains a $\lambda$-dense, isotype subgroup $K$, where $G / K \cong G / H$ and $K$ is not totally projective. Applying our Proposition we construct a $\lambda$-elongation $A$ by $G$, where $p^{\lambda} A$ is a direct sum of cyclic groups of order $p$ and $H$ is maximal in $A$ with respect to having trivial intersection with $p^{\lambda} A$ (see proof of Lemma 1 in $[\mathbf{5}]$ ), that is, $H$ is a so-called $p^{\lambda}$-high subgroup of $A$. Now if $L$ is another $p^{\lambda}$-high subgroup of $A$, then there is a height preserving isomorphism between $L[p]$ and $H[p]$ (see Lemma 6.1 in $[6]$ ) and the generalized Kulikov theorem in [4] implies that $L$ is also totally projective. Thus all $p^{\lambda}$-high subgroups of $A$ are totally projective. Another application of our Proposition yields a second $\lambda$-elongation $B$ by $G$, where $p^{\lambda} B \cong p^{\lambda} A$ and $K$ is a $p^{\lambda}$-high subgroup of $B$. Since $K$ is not totally projective, there can be no isomorphism from $A$ to $B$. 
THEOREM 5. If $G$ is a p-group of countable limit length $\lambda$, then $G$ is totally Zippin if and only if $G$ is a d.s.c.

PROOF. This follows from Theorem 4 by transfinite induction since all countable limit ordinals are cofinal with $\omega$.

\section{REFERENCES}

1. F. Hill, Criteria for total projectivity, Canad. J. Math. 33 (1981), 817-825.

2. P. Hill and C. Megibben, On the congruence of subgroups of totally projectives, Lecture Notes in Math., vol. 1006, Springer-Verlag, Berlin and New York, 1983, pp. 513-518.

3. L. Kulikov, Generalized primary groups. II, Trudy Moskov. Mat. Obšc. 2 (1953), 85-167. (Russian)

4. R. Linton and C. Megibben, Extensions of totally projective groups, Proc. Amer. Math. Soc. 64 (1977), 35-38.

5. C. Megibben, On high subgroups, Pacific. J. Math. 14 (1964), 1353-1358.

6. __ The genealized Kulikov criterion, Canad. J. Math. 21 (1969), 1192-1205.

7. R. Nunke, Homology and direct sums of countable groups, Math. Z. 101 (1967), 182-212.

8. Uniquely elongating modules, Sympos. Math. 13 (1974), 315-330.

9. K. Wallace, On mixed groups of torsion-free rank one with totally projective primary components, J. Algebra 17 (1971), 482-488.

10. $\longrightarrow, C_{\lambda}$-groups and $\lambda$-basic subgroups, Pacific J. Math. 43 (1972), 799-809.

11. R. Warfield, A classification theorem for abelian p-groups, Trans. Amer. Math. Soc. 210 (1975), 149-168.

12. _ The uniqueness of elongations of abelian groups, Pacific J. Math. 52 (1974), 289-304.

13. _ - The structure of mixed abelian groups, Lecture Notes in Math., vol. 616, Springer-Verlag, Berlin and New York, 1977, pp. 1-38.

Department of Mathematics, Vanderbilt University, Nashyille, Tennessee 37235 